\title{
LAZER: UM CONTRIBUTO DA ENFERMAGEM DE REABILITAÇÃO NA AUTONOMIA DA PESSOA COM DPOC
}

\section{LEISURE: A CONTRIBUTION OF REHABILITATION NURSING IN THE AUTONOMY OF PERSON WITH COPD}

\section{OCIO: UNA CONTRIBUCIÓN DE LA ENFERMERÍA DE REHABILITACIÓN EN LA AUTONOMÍA DE LA PERSONA CON EPOC}

DOI 10.33194/rper.2021.179; Data de Receção: 2021-06-18; Data de Aceitação: 2021-10-22

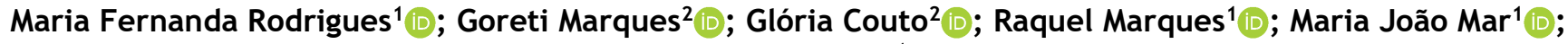 \\ Ana Isabel Araújo' ${ }^{1}$ \\ ${ }^{1}$ Centro Hospitalar Vila Nova de Gaia/Espinho, Portugal; ${ }^{2}$ Escola Superior de Saúde Santa Maria, Porto, Portugal
}

Autor correspondente: Maria Fernanda Rodrigues, nandarpaiva@gmail.com

\section{RESUMO}

Introdução: É amplamente conhecido o impacto das doenças respiratórias na saúde física e mental da pessoa. 0 Enfermeiro Especialista em Enfermagem de Reabilitação sempre foi sensível a esta problemática, sendo foco de intervenção nos seus cuidados a definição de estratégias adaptativas.

Para a pessoa com Doença Pulmonar Obstrutiva Crónica, o contexto atual da situação pandémica trouxe consequências aos vários níveis entre as quais: redução da atividade física, isolamento social e aumento da ansiedade.

Este contexto veio reforçar a importância de uma reflexão acerca da adoção de estratégias adaptativas que promovam a redução da intolerância à atividade da pessoa com Doença Pulmonar Obstrutiva Crónica na execução das Atividades de Vida Diárias nomeadamente as atividades no domínio do "lazer".

Este estudo teve como objetivo avaliar o impacto de um Programa de Reabilitação Respiratória na realização das Atividades de Vida Diárias no domínio do "lazer", medido pela escala London Chest Activity of Daily Living.

Metodologia: Efetuou-se um estudo retrospetivo correlacional, que incluiu utentes com Doença Pulmonar Obstrutiva Crónica admitidos no Programa de Reabilitação Respiratória, sendo os resultados do domínio "Lazer" avaliados, através da escala London Chest Activity of Daily Living, antes e após o Programa de Reabilitação Respiratória. Foram incluídos 46 participantes, (67,39\% do género masculino e 32,6\% do género feminino). A idade dos participantes situou-se entre os 40 e os 89 anos, para uma média de idade de 63 anos $(D P=10,5)$. A maioria dos participantes possui o $1^{\circ}$ ciclo do ensino básico $(52,2 \%)$.

Resultados: Foram identificadas diferenças estatisticamente significativas $(Z=-3,395, p=0,001)$ no domínio do "lazer" da escala London Chest Activity of Daily Living.

Conclusão: Verificaram-se ganhos clínicos no domínio "lazer" da escala London Chest Activity of Daily Living após a realização do Programa de Reabilitação Respiratória.

DESCRITORES: Enfermagem de Reabilitação, Atividades de Vida Diárias, Doença Pulmonar Obstrutiva Crónica, Programa de Reabilitação Respiratória, Lazer.

\section{ABSTRACT}

Introduction: The impact of respiratory diseases on a person's physical and mental health is widely known. The Rehabilitation Nursing Specialist has always been sensitive to this problem, and the focus of intervention in its nursing care plan is the definition of adaptive strategies.

For the person with Chronic Obstructive Pulmonary Disease, the current context of the pandemic situation has brought consequences at various levels, including a reduction in physical activity, social isolation and increased anxiety.

This context reinforced the importance of reflecting on the adoption of strategies that promote the reduction of intolerance to the activity of the person with Chronic Obstructive Pulmonary Disease to carry out Activities of Daily Living, namely in activities in the field of "leisure".

This study aimed to assess the impact of a Respiratory Rehabilitation Program on the realization of Activities of Daily Living in "leisure" domain, measured by the London Chest Activity of Daily Living scale.

Methodology: A retrospective correlational study was carried out, which included patients with Chronic Obstructive Pulmonary Disease admitted to the Respiratory Rehabilitation Program, the results of the Activities of Daily Living in 
the "Leisure" domain was evaluated using the London Chest Activity of Daily Living Scale, before and after the Respiratory Rehabilitation Program. 46 participants were included, (67.39\% male and $32.6 \%$ female), with ages between 40 and 89 years, for an average age of 63 years old $(S D=10.5)$. Most patients had basic level education (52.2\%).

Results: Statistically significant improvements were identified $(Z=-3.395, p=0.001)$ in the "leisure" domain of the London Chest Activity of Daily Living Scale score after Respiratory Rehabilitation Program protocol completion.

Conclusion: There was improvement in the leisure domain of London Chest Activity of Daily Living Scale score after the performance of the Respiratory Rehabilitation Program protocol.

DESCRIPTORS: Rehabilitation Nursing, Daily Living Activities, Chronic Obstructive Pulmonary Disease, Respiratory Rehabilitation Program, Leisure.

\section{RESUMEN}

Introducción: Es ampliamente conocido el impacto de las enfermedades respiratorias en la salud física y mental de los pacientes. El Enfermero Especialista en Enfermería de siempre ha sido sensible a este tema, y parte de su intervención es la definición de estrategias de adaptación.

Para la persona con Enfermedad Pulmonar Obstructiva Crónica, el contexto actual de la situación pandémica ha traído consecuencias a varios niveles, incluida la reducción de la actividad física, el aislamiento social y el aumento de la ansiedad.

Este contexto reforzó la importancia de reflexionar sobre la adopción de estrategias que promuevan la reducción de la intolerancia a la actividad de la persona con Enfermedad Pulmonar Obstructiva Crónica para realizar Actividades de la Vida Diaria, concretamente en actividades del ámbito del "ócio".

Este estudio tuvo como objetivo evaluar el impacto de un Programa de Rehabilitación Respiratoria en la realización de Actividades de la Vida Diaria en el dominio del “ócio", medido por la escala London Chest Activity of Daily Living.

Metodología: Se realizó un estudio correlacional retrospectivo, que incluyó a pacientes con Enfermedad Pulmonar Obstructiva Crónica ingresados en el Programa de Rehabilitación Respiratoria, siendo los resultados del ámbito "ocio" evaluados a través de la Escala London Chest Activity of Daily Living, antes y después del Programa de Rehabilitación Respiratoria. Se incluyeron 46 participantes $(67,39 \%$ hombres y $32,6 \%$ mujeres). La edad de los participantes fue entre 40 y 89 años, con un promedio de edad de 63 años $(D E=10,5)$. La mayoría de los participantes tenía la educación básica (52,2\%).

Resultados: Se identificaron diferencias estadísticamente significativas $(Z=-3.395, p=0.001)$ en el ámbito del "ocio" en la escala London Chest Activity of Daily Living.

Conclusión: Se han comprobado ganancias clínicas en el dominio de "ocio" en la escala London Chest Activity of Daily Livin después de realizar la Programa de Rehabilitación Respiratoria.

DESCRIPTORES: Enfermería de Rehabilitación, Actividades de la Vida Diaria, Enfermedad Pulmonar Obstructiva, Programa de rehabilitación respiratoria crónica, ócio.

\section{INTRODUÇÃO}

À medida que a Doença Pulmonar Obstrutiva Crónica (DPOC) progride, a perda de função pulmonar, as alterações das trocas gasosas e descondicionamento muscular periférico limitam a pessoa nas suas atividades de vida na interação familiar, social e profissional com graves repercussões na qualidade de vida. A incapacidade da pessoa em realizar determinada tarefa ou atividade física, com intensidade ou duração normalmente tolerada por uma pessoa sedentária e sem patologia associada, representa em termos clínicos a intolerância à atividade ${ }^{(1,2)}$. Esta intolerância leva à adoção de estilos de vida mais sedentários na tentativa de evitar a dispneia e a fadiga com o esforço, resultando no agravamento do descondicionamento muscular e consequente diminuição da capacidade funcional levando as pessoas a percecionarem uma menor capacidade para realizar as Atividades de Vida Diárias (AVD), atividade profissional e social, com repercussão na sua condição física, emocional e social. Estas alterações desencadeiam quadros de ansiedade e depressão, conduzindo a sentimentos de tristeza, frustração, alterações do sono, dificuldades de concentração, preocupações somáticas e isolamento social. Neste sentido, a participação na sociedade fica limitada, com perda de qualidade de vida ${ }^{(3,4,5,6,7,8)}$.

A Reabilitação Respiratória é uma intervenção global, multidisciplinar, destinada a pessoas com DPOC, com o objetivo de controlar os sintomas, melhorar a qualidade de vida física e emocional, aumentar a participação social nas atividades quotidianas, bem como diminuir os gastos em saúde mediante a estabilização ou regressão das manifestações da doença ${ }^{(4,9)}$.

Os Programas de Reabilitação Respiratória (PRR) com integração de Enfermeiro Especialista em Enfermagem de Reabilitação (EEER) visam a capacitação da pessoa para melhor gerir a sua doença crónica. No âmbito da sua atividade intervêm em aspetos relacionados com o foco ventilação e intolerância à atividade no sentido de otimizar e melhorar 
a gestão da dispneia e promover a autonomia da pessoa com DPOC para as AVD (10,11,12). Estes programas incluem diferentes componentes: o Treino de Exercício, módulo de educação e apoio psicossocial, suporte nutricional e alteração comportamental $(13,14,15)$.

A solidão e a falta de objetivos de vida associados às alterações patológicas da DPOC, levam a uma deterioração progressiva da qualidade de vida da pessoa. A avaliação do impacto cognitivo, emocional, comportamental e físico da doença nas atividades de vida é de extrema importância, quer na avaliação do estado de saúde, quer na conceção de cuidados de Enfermagem de Reabilitação. Sendo a DPOC uma doença crónica e progressiva, é frequente que a pessoa sinta que não controla a sua evolução, sendo comuns os sintomas de ansiedade e depressão, que podem levar a um maior isolamento social e uma redução da atividade física ${ }^{(16)}$. Por sua vez, pode diminuir a motivação para adquirir conhecimentos e capacidades para o autocontrolo da doença e deste modo, poderá ser afetada negativamente a sua qualidade de vida. Estes são aspetos que tem merecido a atenção dos EEER e são trabalhados pelos mesmos durantes os PRR.

Estas foram as premissas que levaram à elaboração da questão de partida: qual o impacto das atividades desenvolvidas pelo EEER na capacidade da pessoa para realizar as AVD nomeadamente no domínio do "lazer"? Formulamos assim a seguinte hipótese de investigação: as pessoas com DPOC que realizaram o PRR apresentaram menos limitações na realização das AVD no que se refere ao seu "lazer".

O EEER através de intervenções tais como: monitorização/supervisão do treino de exercício, técnicas de Reeducação Funcional Respiratória, controlo ventilatório, gestão de dispneia, capacitação para a gestão e otimização do regime terapêutico e ensino de técnicas de relaxamento para controlo do stress/ansiedade promove a autonomia da pessoa com DPOC nas suas AVD no domínio do “lazer" (4). Visto isto, ter melhor sensação de controlo da sua doença e realizar pequenas atividades de carácter social traduzem melhor qualidade de vida.

A realização deste estudo teve como propósito avaliar o impacto na realização das AVD, no domínio do “lazer”, das pessoas com DPOC submetidas a um PRR. Os objetivos delineados foram: caracterizar as pessoas com DPOC que integraram o PRR e comparar a capacidade dos mesmos para o desempenho nas AVD nomeadamente no domínio do “Lazer".

\section{METODOLOGIA}

Realizou-se um estudo retrospetivo-correlacional de natureza quantitativa, tendo como objetivo avaliar o impacto do PRR nas AVD no domínio do "lazer" nas pessoas com DPOC. No presente trabalho, na avaliação da capacidade da pessoa com DPOC para a realização das AVD recorreu-se à escala London Chest Activity of Daily Living (LCADL) que no domínio do "lazer", avalia aspetos relacionados com a capacidade da pessoa para caminhar dentro de casa, sair socialmente e falar/conversar.

O presente estudo incluiu pessoas com DPOC que integraram o PRR numa Unidade de Reabilitação de um Centro Hospitalar do Norte do País no período entre janeiro e dezembro de 2018. Utilizou-se o processo de amostragem por conveniência em que foram considerados os seguintes critérios de inclusão: pessoas com diagnóstico de DPOC; pessoas que realizaram a totalidade das sessões que integram o PRR e pessoas com avaliação inicial e final completa.

A recolha dos dados foi autorizada pelo Conselho de Administração e pela Comissão de Ética do Centro Hospitalar onde decorreu o estudo (Autorização n 288/2019).

Consideraram-se no decorrer deste estudo os procedimentos éticos necessários. Foi garantido o anonimato e a confidencialidade bem como a exclusividade dos dados. Os dados foram recolhidos numa sala reservada, sendo garantida confidencialidade no manuseamento dos processos e só foram retirados os dados estritamente necessários. Neste sentido, foi criada uma base de dados numerada, sem nunca serem identificados os utentes, e a codificação dos dados foi efetuada unicamente pelo investigador e respetivos colaboradores, sendo assegurado o total sigilo.

$\mathrm{Na}$ análise estatística descritiva utilizaram-se frequências absolutas ( $\mathrm{n}$ ) e relativas (\%); medidas de tendência central: média (M) bem como medidas de dispersão: mínimo (Min), máximo (Max) e desvio padrão (DP). 0 nível de significância (sig) para o tratamento estatístico foi de 5\%. Na análise inferencial foi utilizado o Teste Wilcoxon para amostras emparelhadas.

Por forma a avaliar o nível de limitação das pessoas com DPOC na realização das AVD no domínio do “lazer”, utilizou se como instrumento a escala LCADL aplicado às referidas pessoas, em dois momentos: antes e após o PRR.

O programa realizado pela amostra do presente estudo (quadro 1) foi constituído por 20 sessões, durante 10 semanas com a duração de 90 minutos cada sessão, sendo realizadas 2 sessões por semana em ambiente hospitalar.

Pela importância que o controlo ventilatório assume, nomeadamente quando se progride para o treino ao esforço, as técnicas de reeducação funcional respiratória revelam-se fundamentais para otimizar o utente na sua gestão de energia e definir estratégias para um melhor desempenho das suas AVD. 
Quadro 1 - Caracterização do Programa de Reabilitação

\begin{tabular}{|c|c|c|}
\hline Treino & Tipologia/Modalidade & Tempo/Duração/Intensidade \\
\hline Técnicas de RFR & $\begin{array}{l}\text { Consciencialização e controlo da respiração } \\
\text { Expiração com lábios semicerrados } \\
\text { Reeducação diafragmática posterior } \\
\text { Abertura costal global } \\
\text { Mobilização escapulo-umeral } \\
\text { Reeducação costal global com faixa }\end{array}$ & $\begin{array}{l}15 \text { repetições cada técnica (realizadas na } \\
\text { posição de sentado em frente a espelho } \\
\text { quadriculado) }\end{array}$ \\
\hline Aquecimento & $\begin{array}{l}\text { Exercícios de mobilização global dos membros } \\
\text { superiores e membros inferiores sem carga, para } \\
\text { ativação global dos grupos musculares e } \\
\text { mobilização articular global }\end{array}$ & 5 minutos \\
\hline $\begin{array}{l}\text { Exercício } \\
\text { aeróbio }\end{array}$ & $\begin{array}{l}\text { Bicicleta } \\
\text { Passadeira } \\
\text { Cicloergómetro dos Membros Superiores }\end{array}$ & $\begin{array}{l}20 \text { minutos } \\
10 \text { minutos } \\
\text { Entre } 5 \text { a } 10 \text { minutos } \\
\text { Progressão na intensidade com início a } \\
50 \% \text { até } 80 \% \text { da carga máxima }\end{array}$ \\
\hline $\begin{array}{l}\text { Treino de força } \\
\text { muscular** }\end{array}$ & $\begin{array}{l}\text { Treino com halteres livres, bandas elásticas e } \\
\text { caneleiras } \\
\text { Bíceps e tríceps braquial } \\
\text { Bíceps femoral, abdutores e gémeos } \\
\text { Dorsais e quadríceps (realizados em máquina } \\
\text { dorsais e de quadríceps na posição sentado) }\end{array}$ & $\begin{array}{l}\text { Carga entre } 0,5 \text { e } 3 \text { kg * } \\
10 \text { a } 15 \text { repetições * } \\
1-3 \text { séries * } \\
\text { Duração: } 15 \text { minutos } \\
\text { Carga entre } 2,5 \text { e } 25 \mathrm{w}^{*} \\
10 \text { a } 20 \text { repetições * }\end{array}$ \\
\hline Alongamentos & $\begin{array}{l}\text { Exercícios de alongamento dos principais grupos } \\
\text { musculas dos membros superiores e membros } \\
\text { inferiores }\end{array}$ & 5 minutos \\
\hline
\end{tabular}

*Carga, número de repetições e séries adaptadas à capacidade funcional de cada pessoa bem como a progressão das mesmas ao longo do programa.

**Durante os exercícios que são executados para o treino de força muscular são trabalhados simultaneamente aspetos relacionados com a flexibilidade e o equilíbrio.

O referido programa é composto por várias componentes: Reeducação Funcional Respiratória, Treino de Exercício e sessões de educação para a saúde. O EEER implementa as sessões educacionais, o programa de treino de exercício e efetua ensino e incentivo ao utente para dar continuidade ao programa no domicílio. Para garantir a efetividade do PRR e a adesão ao mesmo, o EEER define estratégias de adaptação para o domicílio, adequadas a cada pessoa de acordo com as suas características individuais e de acordo com os materiais/ equipamentos que cada pessoa possui em casa.

As sessões educacionais realizadas estão evidenciadas na tabela 1.

Tabela 1 - sessões educacionais

Sessões educacionais

Duração/frequência/Metodologia

Fisiopatologia da DPOC

Gestão do regime terapêutico

Técnica inalatória

Técnicas da tosse/relaxamento/ controlo ventilatório

Técnicas de gestão de energia

Cada sessão tem a duração de 45 minutos, de 15 em 15 dias Expositiva/Participativa

Treino de AVD

Adaptação do exercício para o domicílio

Nutrição

\section{RESULTADOS/DISCUSSÃO}

No período de janeiro a dezembro de 2018 foram admitidos para tratamento na unidade de reabilitação, 108 pessoas. Destas, 27 não apresentaram diagnóstico de DPOC, 20 não concluíram o PRR ( 3 por falecimento, 9 por agudização e 8 por motivo desconhecido) e as restantes 15 pessoas não apresentaram dados suficientes na avaliação inicial ou final. 
Após a aplicação dos critérios de inclusão e exclusão, a amostra ficou constituída por 46 pessoas com diagnóstico de DPOC, que integraram o PRR numa unidade de reabilitação de um Centro Hospitalar do Norte do País.

Tabela 2 - Distribuição da amostra segundo as variáveis sociodemográficas.

\begin{tabular}{|c|c|c|c|}
\hline \multicolumn{2}{|c|}{ Variáveis } & \multirow{2}{*}{$\frac{n}{31}$} & \multirow{2}{*}{$\frac{\%}{67,4 \%}$} \\
\hline Género & Masculino & & \\
\hline & Feminino & 15 & $32,6 \%$ \\
\hline \multirow[t]{5}{*}{ Grau de escolaridade } & Sem habilitações & 1 & $2,2 \%$ \\
\hline & Básico $\left(1^{\circ}\right.$ ciclo $)$ & 24 & $52,2 \%$ \\
\hline & Básico $\left(2^{\circ}\right.$ ciclo $)$ & 12 & $26,1 \%$ \\
\hline & Básico ( $3^{\circ}$ ciclo) & 4 & $8,7 \%$ \\
\hline & Secundário & 5 & $10,9 \%$ \\
\hline \multirow[t]{5}{*}{ Idade do utente [anos] } & $40-49$ & 3 & $7 \%$ \\
\hline & $50-59$ & 16 & $35 \%$ \\
\hline & $60-69$ & 14 & $30 \%$ \\
\hline & $70-79$ & 11 & $24 \%$ \\
\hline & $80-89$ & 2 & $4 \%$ \\
\hline
\end{tabular}

A análise das características sociodemográficas (tabela 2) da amostra permite inferir que a maioria dos participantes com diagnóstico de DPOC é do género masculino $(67,39 \%)$ e $32,6 \%$ do género feminino. A idade dos participantes situa-se entre os 40 e os 89 anos, para uma média de idade de 63 anos (DP=10,5). A maioria dos participantes deste estudo apresentam como nível de escolaridade o ensino básico. Os resultados alcançados corroboram outros estudos, particularmente um estudo que pretendia avaliar a confiabilidade do índice de autoeficácia adaptado para reabilitação pulmonar em utentes com patologia respiratória, o qual integrou 150 pacientes com uma idade média de 67 anos. Dos participantes, 46.7\% tinham DPOC e 54\% eram do sexo masculino (17). Num outro estudo, que visava avaliar o impacto da doença respiratória crónica nos autocuidados antes e após o PRR, foi considerada uma amostra de 38 participantes, sendo $71,9 \%$ do sexo masculino com uma idade média de 67 anos ${ }^{(1)}$. Esta evidência foi também obtida na realização de um estudo que pretendia aferir os efeitos de um programa de reabilitação pulmonar na resposta fisiológica e no desempenho das AVD em pessoas com DPOC. Foram incluídas 31 pessoas, na sua maioria homens (71\%), com idade média de 64,2 anos ${ }^{(18)}$.

A idade média dos participantes permite refletir acerca da importância da realização de PRR nesta faixa etária, isto porque são utentes socialmente ativos. É importante o controlo desta doença e investir na capacitação destas pessoas para a gestão da sua doença crónica, aumentando a longevidade e a qualidade de vida. As guidelines têm vindo a reforçar cada vez mais que perante fatores de risco devem ser efetuados diagnósticos precoces, intensificando a relevância da gestão da doença e da atividade física, pelo que é fulcral realizar PRR em faixas etárias mais jovens (19).

No que diz respeito ao nível de escolaridade, no presente estudo $52,2 \%$ da amostra, possuía o $1^{\circ}$ ciclo do ensino básico, 14 participantes concluíram o $2^{\circ}$ ciclo do ensino básico $(26,1 \%)$ e 4 participantes $(8,7 \%)$ frequentaram o $3^{\circ}$ ciclo do ensino básico. O ensino secundário foi frequentado por 5 participantes (10,9\%). Estes achados estão em linha com outro estudo, onde os participantes, maioritariamente $(55 \%)$, frequentaram o ensino básico ${ }^{(20)}$.

O facto de a maioria dos participantes possuir o ensino básico remete para a necessidade de adequar os conteúdos das sessões de educação ao nível de compreensão e conhecimentos dos participantes.

Por forma a comparar a capacidade para o desempenho das AVD das pessoas com DPOC antes e após o PRR, compararam-se os resultados obtidos através da aplicação do instrumento LCADL em dois momentos distintos, antes e após o PRR (tabela 3).

Tabela 3 - Escala LCADL (domínio "lazer") antes e após o PRR

\begin{tabular}{cccccccc}
\hline & & $\mathbf{n}$ & Mínimo & Máximo & Média & DP & Extremos possíveis \\
\hline \multirow{2}{*}{ LCADL } & antes & 46 & 6 & 53 & 25,07 & 10,53 & $0-75$ \\
\cline { 2 - 8 } & após & 46 & 9 & 50 & 19,57 & 9,25 & \\
\multirow{2}{*}{ Domínio “lazer” } & antes & 46 & 0 & 10 & 4,50 & 2,32 & $0-15$ \\
\cline { 2 - 7 } & após & 46 & 0 & 10 & 3,52 & 2,14 & \\
\hline
\end{tabular}


Verifica-se que antes da realização do PRR a média do score total do instrumento LCADL é 25,07 , sendo que o mínimo é 6 e o máximo $53(\mathrm{DP}=10,53)$. Após o PRR, a média do score total é de 19,57 tendo como mínimo um score de 9 e como máximo um score de $50(\mathrm{DP}=9,25)$.

Estes achados revelam uma redução da intolerância na realização das AVD dos participantes, considerando uma diminuição da média dos scores totais de 25,07 para 19,57, antes e após o PRR, respetivamente, sendo que a diferença mínima com significado clínico para a LCADL é de 4 unidades(21), e no presente estudo essa diferença é de 5,5 unidades, considera-se que os resultados obtidos tem significado clínico. Os resultados do presente estudo seguem a evidência de outros estudos. Um deles incluiu 3608 pessoas com diagnóstico de DPOC, sendo o valor do score total da LCADL de 29,6 nas pessoas com DPOC grave e muito grave e 21,4 nas pessoas com DPOC moderada (22). Num outro estudo participaram 60 pessoas com DPOC e também foram demonstradas diferenças estatísticas nos diferentes domínios da escala LCADL ${ }^{(11)}$.

Os resultados obtidos remetem para a importância do papel do EEER na capacitação do utente/ cuidador para as AVD, atenuando as limitações que os utentes ostentam, ajudando na gestão das incapacidades subjacentes à doença crónica.

No domínio “lazer” os participantes referem limitação nas AVD, com um score médio de 4,50. Após o PRR o score neste domínio diminuiu, com a média de 3,52.

Observando a tabela 4, comparando os resultados do domínio “lazer" antes e após o PRR, verificam-se através do teste não paramétrico de Wilcoxon para amostras emparelhadas diferenças estatisticamente significativas $(Z=-3,395$, $p=0,001)$. Verifica-se aumento da autonomia para a realização das AVD no que se refere ao domínio do "lazer", confirmando a hipótese de investigação inicialmente formulada.

Tabela 4 - Teste Wilcoxon para amostras emparelhadas

\begin{tabular}{lcc}
\hline & Z & Sig. \\
\hline Domínio “lazer” após PRR - Domínio “lazer” antes PRR & $-3,395$ & 0,001 \\
\hline LCADL após PRR - LCADL antes PRR & $-4,935$ & $<0,001$ \\
\hline
\end{tabular}

Estes resultados remetem para a importância da valorização das intervenções do EEER durante o PRR nomeadamente a capacitação da pessoa para a realização das AVD através de estratégias adaptativas que traduzem ganhos na capacidade para a execução das mesmas, promovendo a qualidade de vida. Este facto demonstra a importância de incluir no plano as atividades de lazer que a pessoa poderá continuar a realizar e ajudá-la a encontrar estratégias adaptativas para a sua continuidade, para que isto não resulte de um menor investimento da sua parte ou até mesmo um desinvestimento na sua realização levando a um maior isolamento.

O ERR tem um papel central no desenvolvimento e implementação destas terapêuticas, através da conceção, implementação e gestão das terapêuticas que visem o desenvolvimento na pessoa com DPOC de conhecimentos e capacidades para o autocontrolo da doença, e do seu impacto no quotidiano.

\section{CONCLUSÃO}

A finalidade deste estudo consistiu em aferir o impacto do PRR, realizado por EEER nas AVD das pessoas com DPOC nomeadamente no domínio do "lazer" da LCADL. Os resultados do mesmo demonstram ganhos clínicos após a realização do PRR, traduzidos na diminuição das limitações na realização das AVD, por avaliação da escala global.

A realização deste trabalho salienta o papel ativo que o EEER tem nos PRR, contribuindo ao nível da autonomia para as AVD, por um lado, na capacitação para a gestão da doença crónica e definição de estratégias adaptativas para as AVD, e por outro, na implementação dos programas de treino para melhorar a intolerância à atividade, mitigando um conjunto de alterações emocionais, afetivas e educacionais.

Podemos concluir que o PRR que integra este estudo contribui para a diminuição da limitação na realização das AVD, verificando-se melhoria estatisticamente significativa no domínio “lazer” da escala LCADL.

Sugere-se que os PRR tenham continuidade na comunidade onde já existem os PRR com EEER nas Unidades de Cuidados na Comunidade para manter a vigilância e acompanhamento destas pessoas, bem como a realização de estudos que associem a monitorização da tolerância ao exercício.

\section{REFERÊNCIAS BIBLIOGRÁFICAS}

1. Gaspar, L., Martins, P. Reabilitação respiratória em pessoas com bronquiectasias não fibrose quística: qualidade de vida, ansiedade e função respiratória. Rev Port Enf Reab [Internet]. 23 de Junho de 2018 [citado 23 de janeiro de 2021];1(1):38-44. DOI: https://doi.org/10.33194/rper.2018.v1.n1.06.4392

2. Gaspar, L., Martins, P., \& Gomes, F. Efeito da reabilitação respiratória nos sintomas avaliado pelo CAT e a sua relação com a tolerância à 
atividade. Revista Portuguesa de Enfermagem de Reabilitação. Jun de 2019. DPO: https://doi.org/10.33194/rper.2019.v2.n1.01.4566

3. Lottermann P, Sousa C, Liz C. Programas de exercício físico para pessoas com DPOC: uma revisão sistemática. Arq. Cienc. Saúde unipar, Umuarama. 2017; vol. 21, n. 1, 65-75.

4. Gaspar L, Delgado B. Reabilitação respiratória na DPOC: conceitos, componentes, TE in DPOC: abordagem a $360^{\circ}$ do hospital para o domicílio. $1^{\text {a }}$ Ed. Sintra: Lusodidacta Sabooks; 2020. ISBN: 9789895300600.

5. Farias G, Martins R. Qualidade de vida da pessoa com Doença Pulmonar Obstrutiva crónica. Millenium, 45 (jan/jun). 2013; 195-209. Disponível em: https://revistas.rcaap.pt/millenium/article/view/8102

6. Montezinho, S. Reabilitação Pulmonar na DPOC (Tese de mestrado). Coimbra: Faculdade de Medicina da Universidade de Coimbra; 2014.

7. Felício A, Mariz M, Morais A. Vivências da pessoa com Doença Pulmonar Obstrutiva Crónica: Enfermagem de Reabilitação, percursos de investigação. Série monográfica Educação e Investigação em Saúde. Unidade de Investigação em Ciências da Enfermagem: Escola Superior de Enfermagem de Coimbra; 2015. ISSN 1647-9440.

8. Nagamine B, Maciel D. Novos desafios da reabilitação em pacientes DPOC. Research, Society and Development. 2021; vol. 10, n. 4, e10810413901. ISSN 2525-3409 DOI: http://dx.doi.org/10.33448/rsd-v10i4.13901

9. Global iniciative for chronic obstructive disease Global Iniciative for Chronic Obstructive Lung Disease; 2020.

10. Weizenmann V, Pozzobon A. Análise de um programa de reabilitação cardiopulmonar em paciente com doença pulmonar obstrutiva crónicaestudo de caso. Revista destaques académicos. 2014; vol.6, n. ${ }^{\circ 3}$-ccbs/univates.

11. Rocha S. Impacto de um PRR na qualidade de vida e nas atividades de vida diária de pessoas com doença pulmonar obstrutiva crónica (Tese de mestrado). Porto: Escola Superior de Enfermagem do Porto; 2017.

12. Ordem dos Enfermeiros. Guia Orientador de Boa Prática-Reabilitação Respiratória. Ordem dos Enfermeiros. 2018; série 1, Número 10. ISBN 978-989-8444-41-7.

13. Nici L, Donner C, Wouters E, Zuwallack R, Ambrosino N, Bourbeau J. et al. American Thoracic Society/ European respiratory society statement on pulmonar rehabilitation. American jounal of respiratory and critical care medicine. 2006; 173 (12), 1390-1413. DOI: https://doi.org/10.1164/rccm.200508-1211ST

14. Bott J, Blumenthal S, Buxton M, Ellum S, Falconer C, Garrod R, et al. Guidelines for the physiotherapy management of the adult, medical, spontaneously breathing patient - Joint BTS/ACPRC guideline Thorax. 2009; vol. 64, 1-51. DOI: http://dx.doi.org/10.1136/thx.2008.110726

15. Spruit MA, Singh SJ, Garvey C, ZuWallack R, Nici L, Rochester C, et al. An oficial American Thoracic Society/ European respiratory society statement: key concepts and advances in pulmonar rehabilitation. American jounal of respiratory and critical care medicine. 2013 Oct; 188(8): 13-64. DOI: https://doi.org/10.1164/rccm.201309-1634ST

16. Dominic L, Sykes SF, Luke H, Michael G. Impact of COVID-19 on COPD and asthma admissions, and the pandemic from a patient's perspective. Crooks ERJ Open Res 2021; 7: 00822-2020. DOI: https://doi.org/10.1183/23120541.00822-2020

17. Santos, CD., Santos, AJ., Santos, M., Rodrigues, F., Bárbara, C. Pulmonary rehabilitation adapted index of self-efficacy (PRAISE) validated to Portuguese respiratory patients Pulmonology 2019 Nov - Dec; Vol. 25 (6), 334-339.Journal Article. DOI: https://doi.org/10.1016/j.pulmoe.2019.06.003

18. Vaes, A., Delbressine, J., Mesquita, R., Goertz, Y., Janssen, D., Nakken, N.,Franssen, F., Vanfleteren, L., Wouters, E.,Spruit, M.. Impact of pulmonary rehabilitation on activities of daily living in patients with chronic obstructive pulmonary disease Journal of Applied Physiology (2018) Volume 126, 607-615. DOI: https://doi.org/10.1152/japplphysiol.00790.2018

19. Global iniciative for chronic obstructive disease Global Strategy for the Diagnosis, Management, and Prevention of Chronic Obstructive Pulmonary Disease; 2021. DOI: https://doi.org/10.1164/rccm.201204-0596PP

20. Santos, CD., Neves, RC., Ribeiro, RM., Caneiras, C., Rodrigues, F., Spruit, MA., Bárbara, C. Novel Input for Designing Patient-Tailored Pulmonary Rehabilitation: Telemonitoring Physical Activity as a Vital Sign-SMARTREAB Study Journal of clinical medicine [J Clin Med] 2020 Jul 31; Vol. 9 (8). DOI: https://doi.org/10.3390/jcm9082450

21. Direção-geral da saúde (2019). Circular Informativa n 014/2019 “Programas de Reabilitação Respiratória nos Cuidados de Saúde Primários

22. Gonzalez-moro, R., et al. (2009). Impact of COPD severity on physical disability and daily living activities: EDIP-EPOC I and EDIP-EPOC II studies. International Journal Clinical Practice. vol. 63, $\mathrm{n}^{\circ}$ 5, 742-750. DOI: https://doi.org/10.1111/j.1742-1241.2009.02040.x

\section{DIVULGAÇÕES ÉTICAS}

Contribuição do(s) autor(es):

Conceptualização: MFRR; GFSM; GMAC; MJMPC; AICAP; RMRM.

Metodologia: MFRR; GFSM; GMAC; MJMPC; AICAP; RMRM

Validação: MFRR; GFSM; GMAC; MJMPC; AICAP; RMRM.

Análise formal: MFRR; GFSM; GMAC; MJMPC; AICAP; RMRM.

Investigação: MFRR; GFSM; GMAC; MJMPC; AICAP; RMRM.

Tratamento de dados: MFRR; GFSM; GMAC; MJMPC; AICAP; RMRM.

Preparação do rascunho original: MFRR; GFSM; GMAC; MJMPC; AICAP; RMRM.

Redação e edição: MFRR; GFSM; GMAC; MJMPC; AICAP; RMRM.

Revisão: MFRR; GFSM; GMAC; MJMPC; AICAP; RMRM.

Todos os autores leram e concordaram com a versão publicada do manuscrito.

Financiamento:

Este trabalho não recebeu nenhuma contribuição financeira ou bolsa.

Comissão de Ética:

Estudo autorizado pela Comissão de Ética n $288 / 2019$

Declaração de consentimento informado:

Não se aplica.

Agradecimentos:

Os autores agradecem a todos quantos contribuíram para a realização deste trabalho. 
Conflitos de interesse:

Os autores não declaram nenhum conflito de interesses.

Proveniência e revisão por pares:

Não comissionado; revisto externamente por pares.

(C) Autor (es) (ou seu (s) empregador (es)) e APER/RPER 2021. Reutilização permitida de

(c) acordo com CC BY-NC-ND. Nenhuma reutilização comercial. 